\title{
The Tangled Nature Model of evolutionary dynamics reconsidered: structural and dynamical effects of trait inheritance.
}

\author{
Christian Walther Andersen and Paolo Sibani \\ FKF, University of Southern Denmark, DK5230 Odense M, Denmark
}

\begin{abstract}
Based on the stochastic dynamics of interacting agents which reproduce, mutate and die, the Tangled Nature Model describes key emergent features of biological and cultural ecosystems' evolution. While trait inheritance is not included in many applications, i.e. the interactions of an agent and those of its mutated offspring are taken to be uncorrelated, in the family of TNM models introduced in this work correlations of varying strength are parameterised by a positive integer $K$. We first show that the interactions generated by our rule are nearly independent of $K$. Consequently, the structural and dynamical effects of trait inheritance can be studied independently of effects related to the form of the interactions. We then show that changing $K$ strengthens the core structure of the ecology, leads to population abundance distributions better approximated by log-normal probability densities and increases the probability that a species extant at time $t_{\mathrm{w}}$ also survives at $t>t_{\mathrm{w}}$. Finally, survival probabilities of species are shown to decay as powers of the ratio $t / t_{\mathrm{w}}$, a so called pure aging behaviour usually seen in glassy systems of physical origin. We find a quantitative dynamical effect of trait inheritance, namely that increasing the value of $K$ numerically decreases the decay exponent of the species survival probability.
\end{abstract}

PACS numbers: 87.23.-n, 05.40.-a, 87.23.Kg 


\section{INTRODUCTION}

Models of biological [1] and social 2] evolution often involve networks of interacting agents whose dynamics is interpreted in biological or cultural terms [3 5]. Among these, the Tangled Nature Model is a relatively recent [6, 7] but already well-studied [3, 8 15] agent based stochastic description of evolutionary dynamics which features punctuated equilibria [16], a key dynamical property of, among others, macro-evolutionary systems. The latter go through a series of macroscopically different metastable states, with rapid and dramatic changes, here called quakes, leading from one state to the next. Numerical investigations of the TNM show other observed properties of ecosystems, such as the 'area law' 11 and indicate that the model is also applicable to human cultural and industrial ecosystems 14, 15]. As a simple stochastic model the TNM cannot precisely predict the development of an ecosystem from a given initial condition. It can however predict its emergent properties in a variety of different situations and it therefore clearly deserves further development and analysis.

Interestingly, depending on one's choice of how interactions are generated, the TNM can either quickly reach a stationary state characterised e.g. by the power-spectrum of population or diversity fluctuations 3, 12] or it can enter an aging regime [8, 13, 17] similar to that of glassy systems [18]. Specifically [13], a distribution of interactions with finite support leads to stationary behaviour, while aging ensues if ever larger positive interactions become accessible on ever longer time scales. This is the case presently considered, where as seen in [8, 9, 13, 17], the dynamics is driven by a series of non-equilibrium events, our quakes, through a sequence of macroscopically different metastable states, often called QESS (Quasi Evolutionary Stable States).

The set of interactions linking a TNM individual to others is key to its reproductive success and arguably constitutes its most important property. Yet, in many studies, e.g. 8, 13, 17], the interactions of an individual and those of its mutated off-spring are unrelated, a rather unrealistic feature corresponding to a point mutation turning a giraffe into an elephant. To address this issue, Sevim and Rikvold [10] start out with an interaction matrix consisting of Gaussian deviates with variance identical to the uniform distribution previously used [3] by one of these authors. The matrix is then averaged locally over neighborhoods in genome space to produce the desired correlations. These authors find a stationary fluctuation dynamics, resembling that of a model with uncorrelated interactions. We note that their approach requires the storage and manipulation of huge sparse matrices. Laird and Jensen [19] introduce correlated interactions by representing individuals as 16 dimensional vectors in phenotype space. Each element is an integer in the set $\{0,1, \ldots 99999\}$ and contributes additively to the interaction of two species. This leads to a Gaussian interaction distribution where a change of a single element has a minor effect on the total, as desired. The Gaussian distribution of interactions used in both descriptions quickly makes the appearance of 'destabilizers' 13], e.g. mutants receiving very strong positive interactions from extant species, extremely rare, whence the ecology evolves at a pace considerably slower than in Refs. 8, 13, 17. Choosing one type of interaction distribution over another has structural and dynamical effects which seem to have been overshadowed by issues of numerical convenience and, consequently, have not received sufficient attention in the literature.

Below, we introduce and analyze a one-parameter family of TNM models where an increasing degree of correlation between the interactions of an agent and those of its mutated offspring is obtained by increasing the value of a positive integer parameter, $K$, where $K=1$ corresponds the model version without trait inheritance used in Refs. 8, 9, 13, 17]. The interactions between individuals of different species arise as products of two Gaussian variables of zero average. For $K=1$ these are independent and the probability density function (PDF) of the interactions is a modified Bessel function of zeroth order. For $K>1$, the two Gaussian variables independence is no 
longer mathematically guaranteed. Nevertheless, the interactions generated by our rule turn out to be largely insensitive to the value of $K$, and the dynamical effects of trait inheritance can therefore be investigated independently of any effects imputable to a change of the interaction distribution itself.

The rest of the paper is organized as follows: after summarizing the background and notation, we first explain how the interactions are generated, and estimate, for different values of $K$, their distribution and the correlation between the interactions of an agent and those of its mutants. We then describe how trait inheritance affects the dynamics, first qualitatively at the level of the emergent core species structure [13] and then, more quantitatively, in terms of the species abundance distribution and the time decay of a cohort of species picked at different stages of the system evolution.

\section{BACKGROUND AND NOTATION}

TNM agents are binary strings which can either be interpreted as genomes or as cultural features [14, 15], i.e. blueprints or strategies for action. Reproduction is asexual and error prone, and its rate depends on the 'tangle' of interactions connecting the agents, with positive, or mutualistic, interactions leading to a higher reproduction rate. Removals happen at a constant rate and independently of the interactions. Since extant agents draw resources from a shared and finite pool, they all have an indirect, global and negative effect on each other's reproductive success. For sufficiently large values of the coupling probability $\theta$, and irrespective of the degree of correlation, a typical TNM ecology comprises a single group of interacting species.

As described in Ref. [13], the TNM ecology can be sub-divided into a small group of populous core species and a majority of intermittently populated cloud species. The core is an ordered structure, since core species are inevitably linked by mutualistic interactions dynamically selected from a symmetric distribution. The network structure spontaneously emerges in a process driven by an overall increase of configurational entropy [13], showing that entropy and order generation are not necessarily antithetic, as often surmised. In the TNM the two grow simultaneously in different parts of the system: core species carry the order and cloud species carry the entropy. We finally note that the mutualistic interactions between TNM core species do not have a direct interpretation in terms of trophic chains.

For completeness, we now briefly summarize the notation used e.g. in Refs. [8, 13, 17]. A species is a group of agents with identical genomes, and agent ' $a$ ' is queried with probability equal to the relative size of its species. When queried, the agent reproduces with probability

$$
p_{\text {off }}(a)=\frac{1}{1+e^{-H_{a}}}, \quad \text { where } \quad H_{a}=\frac{C}{N(t)}\left(\sum_{i} J_{a i} N_{i}\right)-\mu N_{i} .
$$

In the rightmost expression, from left to right, $C$ is a scaling constant, the coupling $J_{a i}$ represents the influence of agent $i$ on agent $a, N_{i}$ is the current population of species $i$ and $\mu$ is a constant expressing the carrying capacity of the environment. Note that $J_{a i} \neq J_{i a}$ and that $J_{i i}=0$, i.e. selfinteractions are excluded. Each bit (gene) in the genome of a newly created off-spring differs from the parental gene with a constant probability $p_{\text {mut }}$. A last parameter, $\theta$, determines the probability that two species are connected by non-zero interactions. Time $t$ is measured in generations, whose length or duration equals the number $N(t) / p_{\text {kill }}$ of Monte Carlo queries needed on average to remove all individuals present at time $t$ when $p_{\text {kill }}$ is the removal probability. The number of Monte Carlo 
queries within a generation is thus calculated iteratively based on the population of the previous generation.

The parameters used in this work are $\theta=0.25, \mu=0.05, C=50, p_{\text {mut }}=0.01$ and $p_{\text {kill }}=0.25$. Initially, the ecology consists of single species of 500 identical individuals. The above initial condition is standard in the literature and has little bearing on the observed statistical properties on longer time scales. Invariably, the population of a single-and thus non-interacting-species quickly reaches the level dictated by balancing its death and reproduction rates and the evolution of the ecosystem first starts once mutations have created a group of interacting species. In our case, the system is given a couple of generations to find a metastable configuration before data collection begins.

The basic procedure to generate the interactions between species ' $x$ ' and ' $y$ ' from their corresponding binary genomes of length $L, G(x)$ and $G(y)$, relies on both their binary representation and their equivalent integer representation. Right below and until further notice, the name of a species, e.g. $x$, and that of its genome, i.e. $G(x)$, will for convenience be identified in the notation.

Two random arrays of length $2^{L}, A$ and $B$, are initially constructed and never changed during a simulation. $A$ contains independent standard Gaussian deviates of zero average, and $B$ contains independent binary variables which equal zero with probability $1-\theta$ and one with probability $\theta$. The logical exclusive or, $z=\mathrm{XOR}(x, y)$ is a binary string of length $L$ calculated by performing a bitwise XOR operation on the binary representation of $x$ and $y$. The three strings $x, y$ and $z$ are finally read as integer indices to the arrays $A$ and $B$. With an eye to the next section, where the notation is slightly more involved, we denote the $x^{\prime}$ th element of the array $A$ by $A(x)$ instead of the more usual $A_{x}$.

The procedure to generate $J_{x y}$ is now as follows:

1. Calculate $z=\operatorname{XOR}(x, y)$.

2. If $(B(z)=0$ or $x=y)$, set $J_{x y}=0$. If not,

3. (a) Read out the three Gaussian deviates $z_{1}=A(x), z_{2}=A(y)$ and $z_{3}=A(z)$.

(b) Set $J_{x y}=z_{1} z_{3}$ and $J_{y x}=z_{2} z_{3}$.

Note that non-zero interactions are asymmetrical, while zero interactions are symmetrical. Importantly, all interactions are known in potentia before the dynamics starts and never need to be stored in their totality. Computationally this is advantageous, since only the interactions between extant species are needed for updating the dynamical state of the system. These interactions remain at all times a tiny fraction of the existing $2^{2 L}$ possibilities, but move around and acquire more positive values [9, 13, 17] as the ecology evolves.

When using the above scheme, changing a single bit in the genome of an individual leads to a completely different set of interactions to other individuals and to the mentioned lack of trait inheritance. In the next section we illustrate how the scheme can be improved to allow a mutant to inherit some traits of its parent without destroying its computational convenience.

\section{TRAIT INHERITANCE AND CORRELATED INTERACTIONS}

In order to introduce trait inheritance, a gene of length $L$ is first subdivided into $K \leq L$ contiguous parts of integer size $L / K$, each part indexed by an integer $s, 1 \leq s \leq K$. Secondly, we return to our full notation and let $G_{s}(x, 0)$ denote the binary string whose s'th part is identical to the 


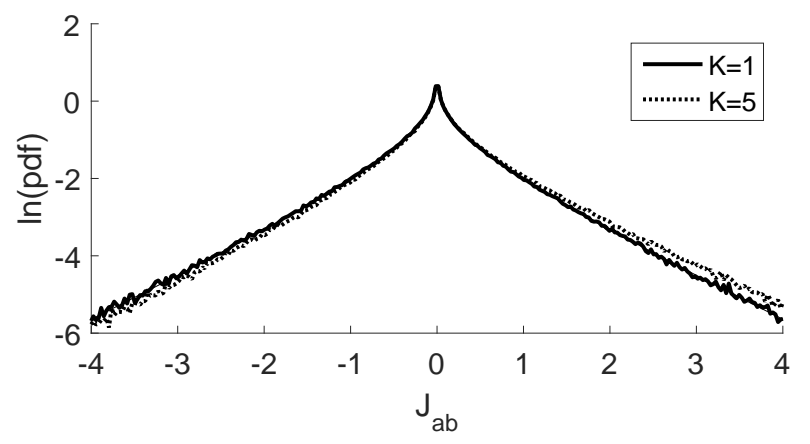

FIG. 1. The PDF of the interaction strengths for two different values of $K$. The theoretical PDF of the product of two standard Gaussian deviates with zero mean is also plotted but is indistinguishable from the the $K=1$ curve at the resolution level of the plot.

corresponding part of $G(x)$, and whose other bits are zero. To calculate the interactions between $x$ and $y$ we repeat the procedure described in the previous section, except that we now read out $3 K$ standard Gaussian deviates, $z_{1, s}=A\left(G_{s}(x, 0)\right), z_{2, s}=A\left(G_{s}(y, 0)\right)$ and $z_{3, s}=A\left(G_{s}(z, 0)\right)$, and define

$$
J_{x y}=z_{1} z_{2} ; J_{y x}=z_{1} z_{3}, \quad \text { where } \quad z_{l}=\frac{1}{\sqrt{ } K} \sum_{s=1}^{K} z_{l, s} ; \quad 1 \leq l \leq 3 .
$$

We note that all three $z_{l}$ 's are standard Gaussian deviates of zero average and that changing one bit in, say, gene $x$ only affects one of the $K$ contributions to $z_{1}$. This produces the desired correlations between the interactions of similar genes. We did not implement an analogous procedure to correlate whether an agent and its one-point mutant have similar sets of zero interactions. Finally, since each part of the genome corresponds to $2^{L / K}$ integers, only $K 2^{L / K}$ elements of the array $A$ are utilized out of the $2^{L}$ available. For $K$ near $L$ this introduces undesired statistical correlations between the $z_{l, s}$ values generated by the algorithm.

The statistical properties of the coupling distribution are summarized in figure 1, which displays an estimate of the PDF of the interaction strengths generated by the rule just described for $L=20$ and for two values of $K: K=1$, corresponding to the uncorrelated model (full line) and $K=5$. For each $K$ value, our estimate of the interaction strength PDF was obtained by generating $10^{4}$ arrays $A$ and $B$. For each of these couples of arrays, $10^{4}$ pairs of species were generated as random binary strings of length $L$. The interactions between each pair were then calculated as explained above. The statistics is thus obtained from $2 \times 10^{8}$ non-zero interactions between random species. Hence it does not include any dynamical selection effects. The theoretical PDF of the product of two standard Gaussian deviates with zero average is also plotted. It does however overlap completely with our empirical $K=1$ curve and cannot be distinguished from it at the resolution level of the plot.

As anticipated, the three PDFs have very similar shapes. The PDF for $K=5$ has a slight positive bias, at very high values of its argument. This is an effect caused, as mentioned, by only using $K 2^{L / K}$ elements of the normally distributed array $A$ : the probability of picking two random numbers with the same sign increases, and when these are multiplied together, the result is always positive. 


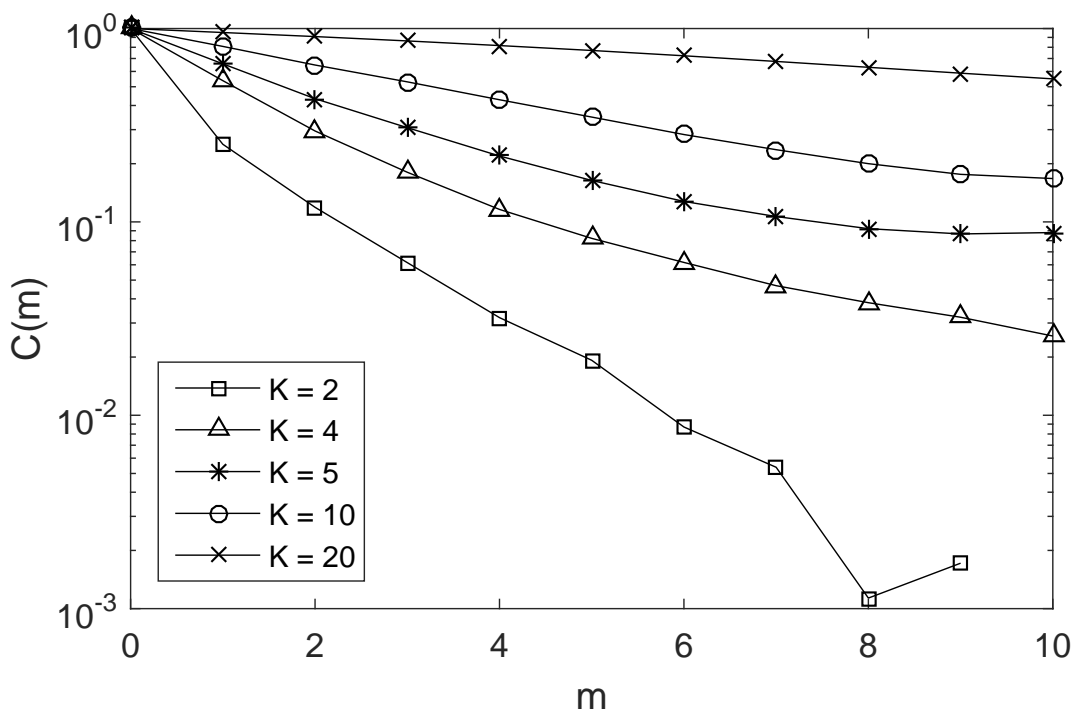

FIG. 2. The normalised correlation function for species pair interactions is plotted on a log scale as a function of the number $m$ of point mutations which one of the two species undergoes.

To describe how the interactions between two agents $a$ and $b$ change as $b$ undergoes mutations, we first pick $10^{4}$ pairs $a, b$ of species and let $J_{a b}(m), m=0,1,2 \ldots$ be the interaction between $a$ and an $m$ times mutated $b$. We then define the correlation function $C(m)=\left\langle J_{a b}(0) J_{a b}(m)\right\rangle_{\{a, b\}}$, where the brackets indicate an average over the different $a$ 's and, for each $a$, over the $\left(\begin{array}{c}L \\ m\end{array}\right)$ possible ways to introduce $m$ point mutations in $b$. For $K=1$ the correlation function normalized to $C(0)=1$ is by construction a Kroneker delta, i.e. $C(m)=\delta_{m 0}$. In fig. 2 similarly normalized correlation functions are plotted on a logarithmic scale as functions of $m$ for several $K$ values above 1 . The functions are seen to decay with $m$ in a nearly exponential fashion and the decay rate is seen to decrease with increasing values of $K$.

In conclusion, our algorithm ensures that a mutant inherits the interactions of its parent. The typical amount of change induced by a mutation decreases with increasing $K$.

\section{DYNAMICAL EFFECTS}

Earlier studies [10, 19] suggest that correlated interactions do not qualitatively change the dynamical behavior of the TNM. In this section we reexamine the question for the model versions considered, focusing on structural features and aging dynamics. Our first analysis concerns the structure of the core species [13] and the log-normal distribution of species abundance. We then proceed to analyze, for different values of $K$, the time dependence of the survival probability of species which are extant at different ages. 

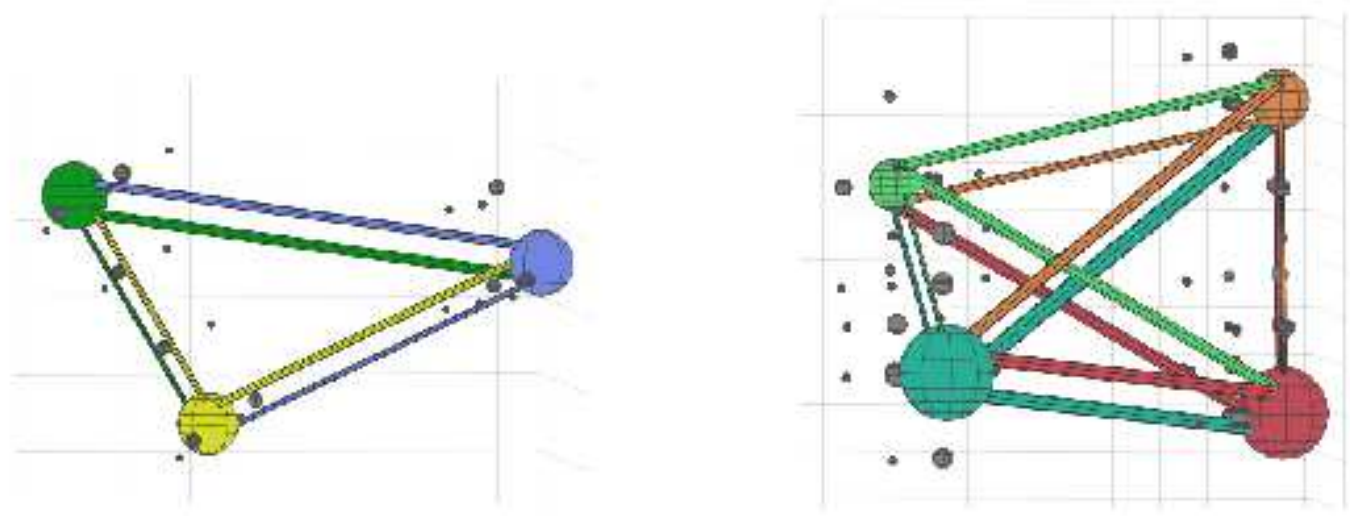

FIG. 3. (Color on line) A 3-dimensional rendering of a single ecology after 10,000 generations. Left, $K=1$ and right $K=5$. Species are represented by spheres of volume proportional to their population. The mutualistic interactions connecting core species are represented by lines with the same color as the species they affect and with a thickness proportional to their strength. Interactions linking cloud species are omitted for clarity.

\section{A. Core structure}

Core species make up the bulk of the population since the mutualistic interactions which link them together endow them with high reproduction rates. Most species belong however to the cloud and are intermittently populated, mainly through an influx of mutants from nearby core species. All together, cloud species only comprise a minor fraction of the population. Following [13], a practical criterion which can be used to distinguish cloud from core species on the fly is that a core species is larger than $5 \%$ of the most populous species.

Figure 3 shows a three dimensional rendering of a single TNM ecology evolved for $10^{4}$ generations starting from a single species with 500 individuals. The mapping is obtained using Principal Component Analysis, see e.g. 14 for further details, and represents species by spheres of volume proportional to their population. Distances reflect the Hamming distances between the corresponding species of the ecology. For $K=5$ the ecology appears more diverse than in the $K=1$ case where interactions are uncorrelated. This seems a natural consequence of the fact that, in the former case, a mutant species inherits to some degree the good connections of its parent core species and can more easily establish itself as a new core species. This seems to be the case for the two nearby core species drawn near the lower edge of the right panel. Besides one extra core species, the $K=5$ version features many more cloud species and a larger total population. To show in more detail the differences in population and diversity induced by changing $K$, simulations were continued for a few generations after forcing the ecology to 'freeze' at $t=10^{4}$, by instantanuosly setting the mutation rate $\mu_{\text {mut }}$ to zero. The immediate effect of removing mutations, seen in both panels of Fig. 4 , is that core species grow in size, due to the fact that all cloud species, no longer replenished by mutants, die out. The long term effect is to force the dynamics into a stationary state by preventing the generation of destabilising mutants. Figure 4 depicts core species populations in such state for $K=1$ and $K=5$ as a function of time and shows that turning on the correlations 

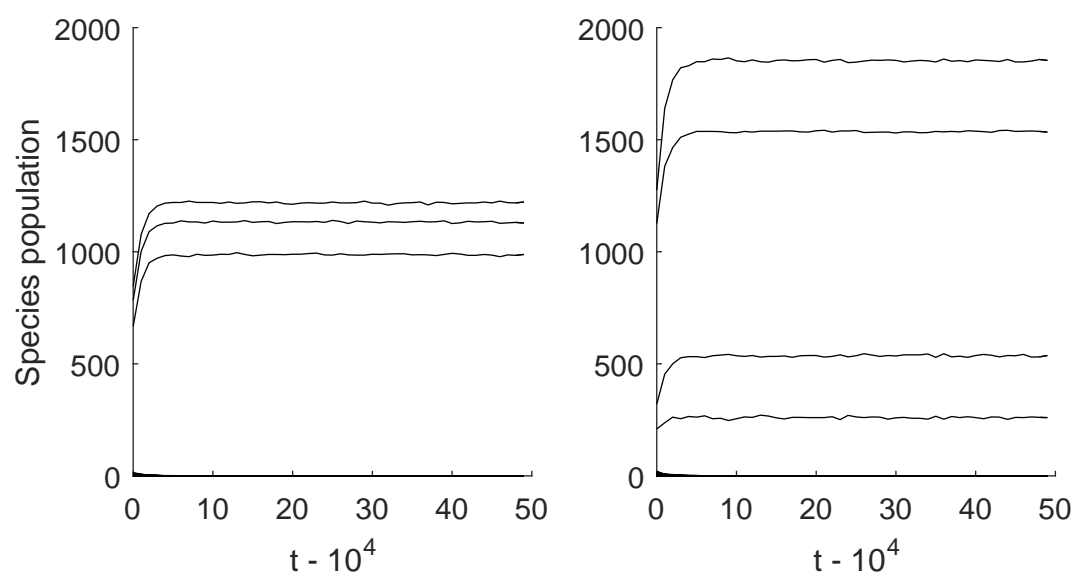

FIG. 4. Each line represents the population of a different species in the frozen ecology obtained by instantanuosly setting the mutation rate to zero after $10^{4}$ generations. The left and right panels show uncorrelated and correlated interactions, respectively.
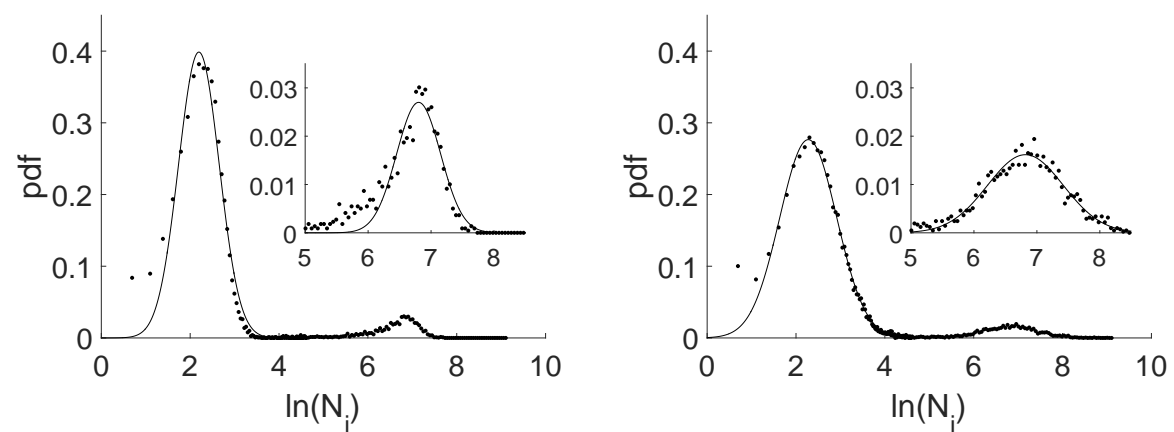

FIG. 5. The population abundance distributions vs. the logarithm of the species population for $K=1$ (left) and $K=5$ (right), respectively. Lines are gaussian fits. Notice the clear separation on the horizontal scale of the two maxima of the distribution. Insets zoom in on core species data at large populations.

increases both the diversity and the total population of the ecology.

\section{B. Population abundance distribution}

Figure 5 illustrates how the population is distributed across different species. The abscissa is the logarithm of the species population size, and the ordinate is our estimate of the corresponding probability distribution function, obtained as the frequency with which a species of given size appears in the population. The data were obtained from an ensemble of 100 independent runs each lasting for $10^{4}$ generations. Comparing the left and right panels, we note that, irrespective of $K$, the abundance distribution is bimodal, with two widely separated maxima corresponding to 
values of the abscissa near 2 and 7. The separation supports the distinction between cloud and core species. The insets show that, for $K=5$, core species are distributed in a nearly log-normal fashion while, for $K=1$, a log-normal distribution still provides a reasonable, but far less accurate, description. We also see that the distribution of the most populous half of the cloud species is also approximately log-normal, while very sparsely populated species fall outside the description. The nearly log-normal distribution of cloud species is expected, as these species are populated by an influx of mutants from 'parent' core species with the size of the influx mirroring the size of the parent species.

Anderson and Jensen [9] already pointed out the relevance for the TNM of log-normal distributions, which are known to describe many natural systems, including abundance distributions in several real ecologies [20]. Our results concur with theirs and furthermore show $i$ ) that the distinction between core and cloud species emerges naturally from a statistical analysis and $i i$ ) that introducing correlations improves the quality of the log-normal description of the species abundance distribution.

\section{SPECIES PERSISTENCE CURVES}

Since, by definition, TNM agents die at a constant rate, they have a finite expected life-time. In contrast, and reflecting the complexity of the dynamics, species die at a slow and decelerating rate, and their life-times do not possess finite averages. Studying species persistence curves throws light on important aspect of the TNM dynamics: first, these curves concisely describe the aging dynamics of the model and, second, they change in a measurable and systematic way when inherited traits are introduced.

To explore these issues we define a cohort as the set of species extant at time $t_{w}$. We then measure the persistence $P\left(t_{w}, t\right)$ as the fraction of the cohort which is still extant at time $t>t_{w}$. The persistence provides an estimate of the probability that a species extant at time $t_{w}$ still is extant at later times and the distinction between the two quantities is glossed over in the following. The life-time probability density function of a species extant at $t_{w}$ is then

$$
S\left(t_{w}, \tau\right)=-\frac{d}{d \tau} P\left(t_{w}, t_{w}+\tau\right) \quad 0 \leq \tau<\infty .
$$

To measure persistence of species we ran 51 independent simulations in the $K=1$ case and 68 in the $K=5$ case, all lasting $2^{24}>10^{7}$ generations, which is a very long time by most criteria. Extant species are registered at each $t=2^{k}, k=4,5 \ldots 24$, with the delay on the first cohort introduced to ensure that the ecology has properly stabilised. We thus end up with 21 cohorts, one for each $k$, of which the last one is discarded as it only has one data point. Each data set in figure 6 shows for $t>t_{w}$ the persistence of the cohort of species extant at time $t_{w}$. The dip for $t \approx t_{w}$ stems from the fast disappearance of cloud species. At later times the curves all tend to approach straight lines on our log-log plot, which is indicative of a power-law dependence. Since pure or $t / t_{w}$ scaling is known to hold approximately in many aging systems of physical nature, see Ref. 21] and references therein, it is interesting to investigate its applicability to a model of biological evolution such as the TNM. Figure 7 shows that plotting our persistence data as a function of $t / t_{w}$ produces a good data collapse. The lines are fits to a power-law $y\left(t / t_{w}\right)=a\left(t / t_{w}\right)^{b}$. For $K=1$ the exponent is $b=-0.283(14)$ and for $K=5$ it is $b=-0.117(6) . z$ Three comments are in order: first, independently of the degree of inheritance, Eq. (3) shows that the life-time distribution lacks a finite average. Second, we see that species created at a late stage of the evolution process (large $t_{w}$ ) are more resilient than those created early on, implying that the rate of quakes decreases in time. Third, the exponent of the 


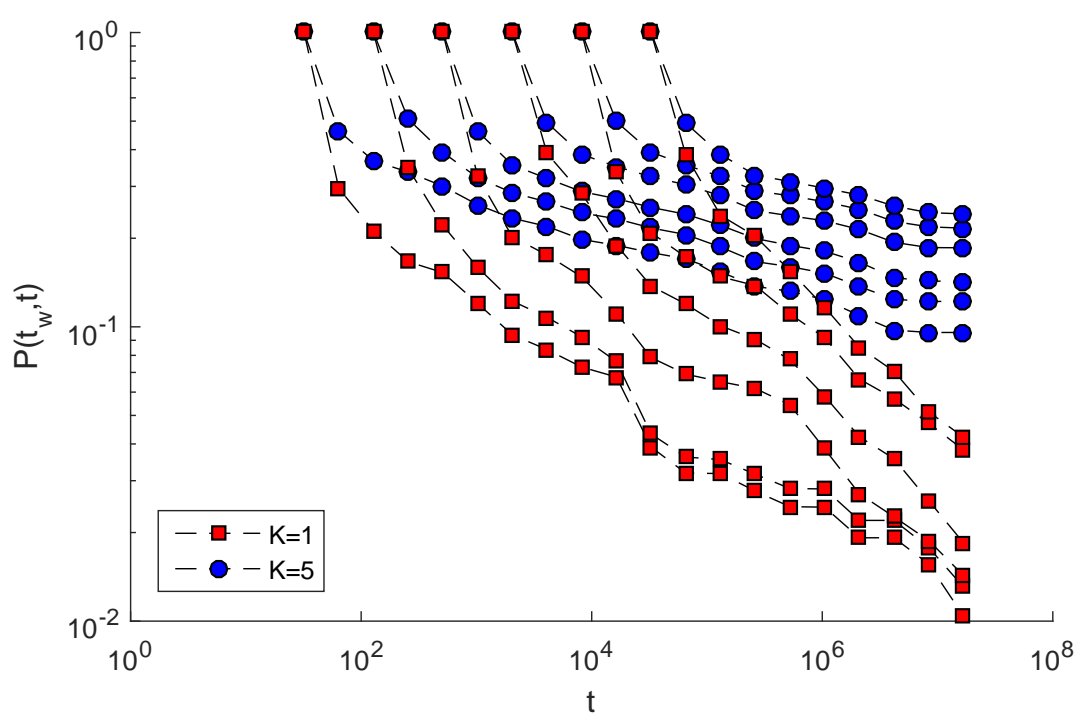

FIG. 6. (Color on line) Species persistence versus time. A new cohort of extant species is registered for each $t=2^{k}, k=1,2,3, \ldots$, and the corresponding fraction surviving at later times is plotted on a logarithmic scale. Not all cohorts are shown for graphical reasons. $K=1$ data are shown by squares, and $K=5$ data by circles. Lines are only guides to the eye.

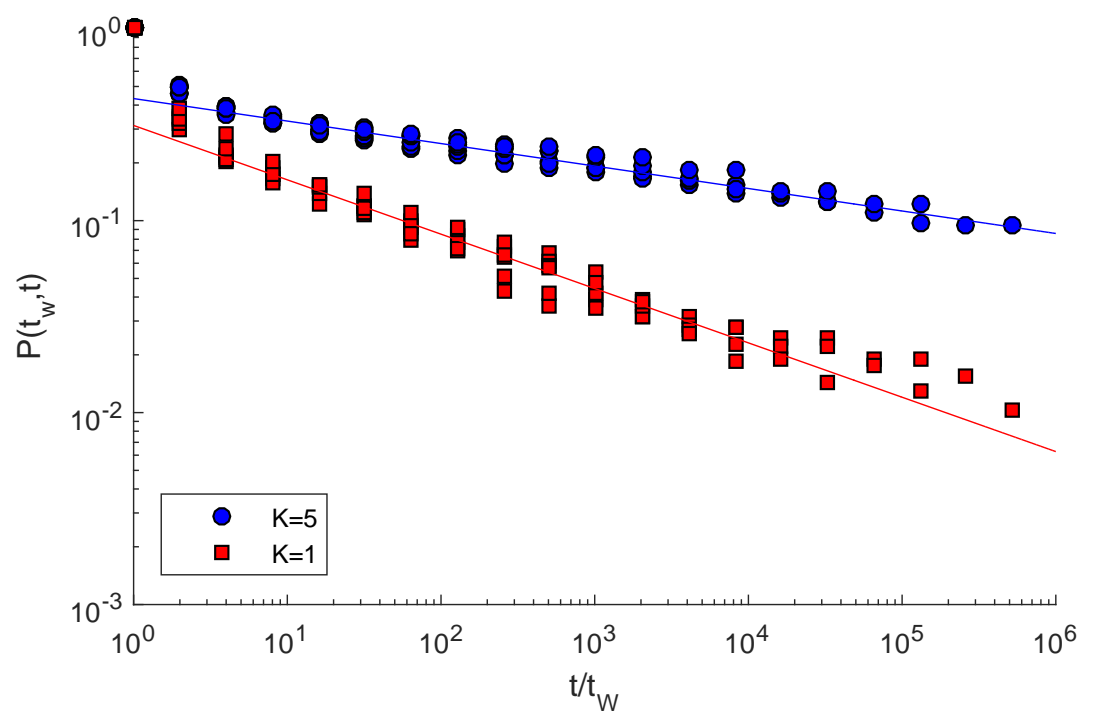

FIG. 7. (Color on line) same data as in figure 6, now plotted as a function of $t / t_{w} . K=1$ data are shown by squares, and $K=5$ data by circles. The lines are least squares fits to power-laws $y=a\left(t / t_{w}\right)^{b}$. where $b=-0.283(14)$ and $b=-0.117(6)$ for $K=1$ and $K=5$, respectively. All available cohorts have been used in the fits. 
persistence decay is more than halved when $K$ goes from 1 to 5 , clearly showing that inheritance produces a more robust ecology where species live longer.

\section{CONCLUSION AND OUTLOOK}

In the TNM version introduced in this work, a mutant inherits part of the interactions of its parent, with the amount of modification controlled by an integer parameter $K$. Independently of $K$, the genome remains a point in an $L$ dimensional hypercube, and the value of $K$ has only insignificant effects of the distribution of the non-zero couplings linking different species. Finally, our approach does not require the storage and manipulation of huge sparse matrices. We concur with Refs. 10, 19] that trait inheritance does not radically changes the basic properties of the TNM: irrespective of the value of $K$, we see a decelerating aging dynamics where sudden quakes lead to considerable rearrangements of the network structure of the TNM ecology. More specifically, independently of $K$ the aging dynamics of the TNM is characterized by persistence curves which decay as powers of the ratio $t / t_{w}$, a scaling form known as pure aging in complex systems of physical origin [18]. Increasing the degree of inheritance has nevertheless both structural and dynamical effects: The sub-net of core species becomes larger and more highly populated, the species abundance distribution is better approximated by the log-normal distribution found in many experimental data, and the decay of the species survival probability becomes noticeably slower.

Trait inheritance generates TNM core species which are related to each other. In real ecologies, be they of biological or socio-economic nature, families of related extant species are the norm rather then the exception, and the selective pressure generated a varying external parameter is more likely to change the relative population of these related species than to destroy the ecosystem itself. We expect the added flexibility to external variations to be a property of the TNM with trait inheritance which is worth of further investigations.

\section{ACKNOWLEDGMENTS}

PS would like to thank Peter Salamon for motivating part of this work and Rudy Arthur for several stimulating discussions on TNM dynamics.

[1] Barbara Drossel. Biological evolution and statistical physics. Advances in physics, 50:209-295, 2001.

[2] Claudio Castellano, Santo Fortunato, and Vittorio Loreto. Statistical physics of social dynamics. Reviews of Modern Physics, 81:591-646, 2009.

[3] Per Arne Rikvold and R. K. P. Zia. Punctuated equilibria and $1 / f$ noise in a biological coevolution model with individual-based dynamics. Phys. Rev. E, 68:031913, 2003.

[4] R. Axelrod. The dissemination of culture: A model with local convergence and global polarization. Journal of Conflict Resolution, 41(3):203-226, 1997.

[5] Marco Alberto Javarone and Giuliano Armano. Perception of similarity: a model for social network dynamics. Journal of Physics A, 46(45): 455102, 2013.

[6] K. Christensen, S.A. de Collobiano, M. Hall, and H.J. Jensen. Tangled nature: A model of evolutionary ecology. Journal of Theoretical Biology, 216(1):73-84, 2002.

[7] Matt Hall, Kim Christensen, Simone A. di Collobiano, and Henrik Jeldtoft Jensen. Time-dependent 
extinction rate and species abundance in a tangled-nature model of biological evolution. Phys. Rev. E, 66:011904, 2002.

[8] Paul Anderson, Henrik Jeldtoft Jensen, L.P. Oliveira and Paolo Sibani. Evolution in complex systems. Complexity, 10:49-56, 2004.

[9] PE Anderson and HJ Jensen. Network properties, species abundance and evolution in a model of evolutionary ecology. Journal of Theoretical Biology, 232:551-558, 2005.

[10] Volkan Sevim and Per Arne Rikvold. Effects of correlated interactions in a biological coevolution model with individual-based dynamics. Journal of Physics A, 2005.

[11] D. Lawson and H. J. Jensen. The species-area relationship and evolution. Journal of Theoretical Biology, 241(3):590-600, 2006. Cited By (since 1996):9.

[12] Yoshuke Murase, Takashi Shimada, Nobuyasu Ito, and Per Arne Rikvold. Random walk in genome space: A key ingredient of intermittent dynamics of community assembly on evolutionary time scales. Journal of Theoretical Biology, 264:663-672, 2010.

[13] Nikolaj Becker and Paolo Sibani. Evolution and non-equilibrium physics: A study of the Tangled Nature Model. EPL, 105:18005, 2014.

[14] A.E Nicholson and P Sibani. Cultural evolution as a nonstationary stochastic process. Complexity, 2015.

[15] Rudy Arthur, Arwen Nicholson, Paolo Sibani and Michael Christensen. The Tangled Nature Model for Organizational Ecology. Computational and Mathematical Organization Theory, 2016.

[16] Stephen Jay Gould and Niles Eldredge. Computational and Mathematical Organization Theory, 2016. Punctuated equilibria: the tempo and mode of evolution reconsidered. Paleobiology 3 (2): 115-151, 1977.

[17] Dominic Jones, Henrik Jeldtoft Jensen, and Paolo Sibani. Tempo and mode of evolution in the tangled nature model. Physical Review E, 82, 2010.

[18] Paolo Sibani and Henrik Jeldtoft Jensen . Stochastic Dynamics of Complex Systems: From Glasses to Evolution. Imperial College Press, 2014.

[19] Simon Laird and Henrik Jeldtoft Jensen. The Tangled Nature model with inheritance and constraint: Evolutionary ecology restricted by a conserved resource. Ecological Complexity, 3(3):253-262, SEP 2006.

[20] Eckhard Limpert, Werner A. Stahel, and Markus Abbt. Log-normal distributions across the sciences: Keys and clues. Bioscience, 51(5):341-352, 2001.

[21] Paolo Sibani and Gregory G. Kenning. Origin of 'end of aging' and sub-aging scaling behavior in glassy dynamics. Phys. Rev. E, 81:011108, 2010. 